\title{
The burden of managing pleural effusions in patients with chronic myelogenous leukemia post-imatinib failure: A literature-based economic analysis
}

This article was published in the following Dove Press journal:

International Journal of General Medicine

21 December 2009

Number of times this article has been viewed

Jennifer Stephens

Kimbach Tran Carpiuc

Marc Botteman

Pharmerit North America

LLC, Bethesda, MD, USA
Correspondence: Jennifer M Stephens Clinical Director, Pharmerit North America LLC, 4350 East-West Highway, Suite 430, Bethesda, MD 208I4, USA

$\mathrm{Tel}+|24082| 1290$

Fax +I 240 82I I296

Email jstephens@pharmerit.com
Objectives: To develop an economic analysis of the management of pleural effusions in patients with imatinib-resistant/intolerant chronic myelogenous leukemia (CML).

Methods: A cost of treatment analysis was conducted from the US payer perspective, based on resource utilization data for 48 patients with dasatinib-related pleural effusions at a large US cancer center. Probabilities of various procedures and treatment events were derived from published resource use data, supplemented with expert opinion. Cost data was derived from median reimbursements for relevant CPT codes for outpatient services and medical literature for inpatient services. Sensitivity analyses were conducted for types of procedures used. All costs were adjusted to US dollars (2007 rates).

Results: Sixty percent of pleural effusions were managed medically costing \$750 per episode. Forty percent of pleural effusions were more significant ( $>25 \%$ of one lung volume), with half of those requiring invasive procedures. Cost of inpatient procedures was $\$ 10,616$ for chest tube and $\$ 15,170$ with pleural catheter. Cost of outpatient procedures was $\$ 713$ for ultrasound thoracentesis and $\$ 4,598$ for pleural catheter. The average cost of treating a pleural effusion was $\$ 2,062$ to $>\$ 2,700$ for all severity levels and $\sim \$ 6,400$ to $>\$ 9,000$ for invasive procedures. Key cost drivers were invasive procedures and recurrence.

Conclusion: This economic analysis using actually observed treatment patterns suggests that the management of pleural effusion adverse events in CML patients is costly, requires intensive resource utilization, and may be an important factor in treatment selection.

Keywords: dasatinib, nilotinib, imatinib, adverse events, safety, cost, and cost analysis

\section{Introduction}

Imatinib has revolutionized the treatment of chronic myelogenous leukemia (CML) as targeted therapy acting on the abnormal protein Bcr-Abl produced by the Philadelphia chromosome. With five-year survival rates of $89 \%$ in chronic phase CML (CML-CP), imatinib is the first-line standard of care for CML-CP treatment. ${ }^{1}$ However, some CML patients may become resistant to imatinib or do not tolerate imatinib.

Two new drugs targeted for CML, nilotinib and dasatinib, have been developed as options for imatinib-resistant and/or -intolerant patients. Although these newer drugs are effective in obtaining responses in imatinib-resistant/intolerant CML, their target ${ }^{2}$ and adverse event profiles differ. ${ }^{3,4}$

Pleural effusion is usually a rare drug-related adverse event, typically resulting in the need for some type of intervention. Common symptoms of pleural effusion include significant cough, fatigue, and dyspnea, which may affect the patient's quality of life. ${ }^{5}$ Risk of effusions exists with all the tyrosine kinase inhibitors (TKIs) currently indicated 
for CML (imatinib, dasatinib, and nilotinib), but is most commonly seen with dasatinib. Recent data from imatinibresistant patients receiving dasatinib at a large cancer center show that pleural effusion events occur in up to $35 \%$ of patients, with risk persisting over time. ${ }^{6}$ Pleural effusion may emerge unexpectedly and as late as 24 months into therapy with few predictive factors. These effusions can lead to other complications and require additional medical resource use beyond the typical routine care. ${ }^{6,7}$ The interventions may be costly and add to the overall economic burden and resource use of managing CML patients.

Given the published clinical and resource use details provided for pleural effusion management in dasatinib-treated patients, ${ }^{6,7}$ our objective was to develop an economic burden analysis of treating pleural effusions in CML patients by applying costs to the published medical resource utilization rates.

\section{Methods}

We developed a literature-based cost-of-treatment analysis to determine the economic burden associated with treating pleural effusions in CML patients with imatinib resistance/intolerance. The analysis adopted a US third party payer/insurer perspective including both inpatient and outpatient direct medical costs. The model provides cost-of-treatment estimates for managing pleural effusion adverse events, from initiation of therapy to two years of follow-up. The primary clinical input data were derived from the observed medical resource utilization reported for 48 patients with dasatinib-related pleural effusions at one large cancer center. ${ }^{6,7}$

Table 1 summarizes the treatment patterns and rates of resource utilization associated with dasatinib-related pleural effusions from MD Anderson Cancer Center: 6,7 38\% received an echocardiogram $(\mathrm{ECHO}) ; 71 \%$ received a course of diuretics; $29 \%$ had recurrent effusions; 30\% received steroids; $19 \%$ required a median of 3 (range 1-12) thoracentesis outpatient procedures (or outpatient pleural catheter placement) due to dyspnea grade $\geq 3$ with median volume withdrawn per thoracentesis of $1.5 \mathrm{~L}$ [range $0.5-2 \mathrm{~L}$ ]); $4 \%$ were managed with inpatient chest tube or pleural catheter

Table I Clinical and resource use management of pleural effusions in $\mathrm{CML}^{6,7}$

\begin{tabular}{|c|c|}
\hline Severity & Reported management of dasatinib pleural effusion \\
\hline All Levels & $\begin{array}{l}\text { - Physician evaluation } \\
\text { - ECHO (38\%); and some CT scans (\% not specified) } \\
\text { - Chest X-ray (I00\%) } \\
\text { - Dose interruptions in } 83 \% \text { for median of } 27 \text { days (range } 4-113 \text { days), with more than } \\
\text { one interruption in } 29 \% \text { for recurrence } \\
\text { - Dose reduction in } 71 \%(n=34)\end{array}$ \\
\hline $\begin{array}{l}\text { Level } \mathrm{I}-2 \\
\text { (up to } 25 \% \text { of one lung volume) }\end{array}$ & $\begin{array}{l}\text { - All episodes resolved upon } \\
\text { *dasatinib continuation ( } 28 \%) \\
\text { *loop diuretics }(14 \%) \\
\text { *both loop diuretics and discontinuation }(55 \%) \\
\text { - A short course of oral prednisone }(40 \mathrm{mg} / \text { day } \times 4) \text { was required in } 24 \% \text { of low-grade } \\
\text { effusion patients, which resulted in disappearance of effusion in }<72 \text { hours } \\
\text { - Invasive procedures in } 7 \% \text { (both level } 2 \text { ) }\end{array}$ \\
\hline $\begin{array}{l}\text { Level } 3 \\
\text { ( } 26 \%-50 \% \text { of one lung volume) }\end{array}$ & $\begin{array}{l}\text { - } 81 \% \text { interrupted dasatinib and received loop diuretics (the others continued dasatinib } \\
\text { due to advanced disease) } \\
\text { - } 44 \% \text { received a short course of oral prednisone } \\
\text { - Invasive procedures in } 25 \%\end{array}$ \\
\hline $\begin{array}{l}\text { Level } 4-5 \\
\text { ( } 5 \text { I to }>75 \% \text { of one lung volume) }\end{array}$ & - All interrupted dasatinib and required invasive procedures for management \\
\hline Invasive procedures & $\begin{array}{l}\text { - } 19 \% \text { of all patients with pleural effusions required a median of three thoracenteses } \\
\text { (range } \mathrm{I}-12 \text { thoracenteses per patient due to dyspnea grade } \geq 3 \text {; median volume } \\
\text { withdrawn per thoracentesis was } \mathrm{I} .5 \mathrm{~L} \text { [range } 0.5-2 \mathrm{~L}] \text { ) } \\
\text { - } 4 \% \text { of all patients required placement of chest tube } \\
\text { - } 4 \% \text { of all patients required placement of a Denver peritoneovenous shunt } \\
\text { - } 2 \% \text { of patients received a pericardial window }\end{array}$ \\
\hline
\end{tabular}

Notes: Treatment categorized by level of effusion (not $\mathrm{NCl}$ grade) and based on volume of lung involved. 
placement; $4 \%$ required Denver shunts as inpatients for recurrent effusions; and $2 \%$ required an inpatient pericardial window (for pericardial effusion coexisting with the pleural effusion).

Clinical expert input was used only to supplement the literature related to assumptions of frequency of office visits and chest X-rays. For this economic analysis, we assumed all patients would incur two additional physician office visits for diagnosis and follow-up of pleural effusion, and that two chest $\mathrm{X}$-rays (one at each visit) would be performed. This was done to account for a real-world practice pattern that was not reflected in dasatinib pleural effusion management at the cancer center, given that the patient follow-up visits were protocol driven during the study.

Standard costs for relevant CPT codes and median reimbursement fees for outpatient procedures and office visits were retrieved from the Ingenix National Fee Analyzer. ${ }^{8,9}$ The CPT code for placement of a Denver peritoneovenous shunt (used for a recurrent effusion) was not available. The estimated cost for this procedure was based on the CPT code 49425 , insertion of a peritoneal venous shunt, and added costs for associated care: two days of general ward hospital stay, and two days of physician inpatient visits.

The cost of inpatient management of pleural effusions with chest tube placement and other invasive procedures were obtained from the medical literature. ${ }^{10,11}$ The cost of inpatient chest tube placement was $\$ 10,616$, while an inpatient pleural catheter placement was $\$ 15,170$ based on details provided by Putnam and colleagues. ${ }^{10}$ The cost of an inpatient pericardial window was $\$ 15,344$ based on details provided by Girardi and colleagues. ${ }^{11}$

Drug costs for medical management with diuretics (furosemide $40 \mathrm{mg}$ daily for 10 days) and steroids (oral prednisone $40 \mathrm{mg}$ daily for four days) were based on generic cost data from the Redbook. ${ }^{12}$ In addition to the drug acquisition costs of less than one dollar for a course of either generic furosemide or prednisone, we included a standard \$7 dispensing fee to more accurately reflect the true cost of having the medication filled in an outpatient pharmacy.

Table 2 lists the CPT code, drug, and literature costs that were included in our analysis, along with associated probabilities used in calculating the cost of pleural effusions.

Table 2 Results of economic analysis presenting average cost for treatment of a pleural effusion in CML

\begin{tabular}{|c|c|c|c|c|c|c|}
\hline Health care resource use & $\begin{array}{l}\text { Unit cost per } \\
\text { events }\end{array}$ & $\begin{array}{l}\text { Number } \\
\text { of units }\end{array}$ & Probability & $\begin{array}{l}\text { Invasive } \\
\text { procedure } \\
\text { subgroup } \\
\text { probabilities }\end{array}$ & $\begin{array}{l}\text { Estimated avg } \\
\text { cost of treatment } \\
\text { per CML patient } \\
\text { across all effusions }\end{array}$ & $\begin{array}{l}\text { Estimated avg cost } \\
\text { of treatment per } \\
\text { patient requiring } \\
\text { invasive procedures }\end{array}$ \\
\hline Course of diuretics & $\$ 8$ & 1.29 & 0.68 & 1.00 & $\$ 7$ & $\$ 10$ \\
\hline Short course of steroids & $\$ 8$ & 1.29 & 0.29 & 0.58 & $\$ 3$ & $\$ 6$ \\
\hline Chest X-ray (2 views; CPT 7I020) & $\$ 100$ & 2.58 & 1.00 & 1.00 & $\$ 258$ & $\$ 258$ \\
\hline ECHO (CPT 93307) & $\$ 484$ & 1 & 0.38 & 1.00 & $\$ 182$ & $\$ 484$ \\
\hline Physician office visits (CPT 992।4) & $\$ 117$ & 2.58 & 1.00 & 1.00 & $\$ 301$ & $\$ 301$ \\
\hline $\begin{array}{l}\text { Outpatient ultrasound thoracentesis } \\
\text { (CPT 32000) or }\end{array}$ & $\$ 713$ & 3 & 0.19 & 0.75 & $\$ 406$ & $\begin{array}{l}\$ 1,604 \\
\text { or }\end{array}$ \\
\hline Outpatient pleural catheter placement ${ }^{10}$ & $\$ 4,598$ & 1 & 0.19 & 0.75 & $\$ 874$ & $\$ 3,449$ \\
\hline $\begin{array}{l}\text { Inpatient chest tube placement }{ }^{10} \\
\text { or }\end{array}$ & $\$ 10,616$ & 1 & 0.04 & 0.17 & $\$ 446$ & $\begin{array}{l}\$ 1,805 \\
\text { or }\end{array}$ \\
\hline Inpatient pleural catheter placement ${ }^{10}$ & $\$ 15,170$ & 1 & 0.04 & 0.17 & $\$ 637$ & $\$ 2,579$ \\
\hline Denver shunt placement* & $\$ 3,807$ & 1 & 0.04 & 0.17 & $\$ 152$ & $\$ 647$ \\
\hline Inpatient pericardial window" & $\$ 15,344$ & 1 & 0.02 & 0.08 & $\$ 307$ & $\$ 1,279$ \\
\hline & & \multicolumn{3}{|c|}{$\begin{array}{l}\text { Average per patient cost without } \\
\text { pleural catheter: }\end{array}$} & $\$ 2,062$ & $\$ 6,394$ \\
\hline & & \multicolumn{3}{|c|}{$\begin{array}{l}\text { Average per patient cost with } \\
\text { pleural catheter: }\end{array}$} & $\$ 2,7 \mid 7$ & $\$ 9,013$ \\
\hline
\end{tabular}

Notes: Costs in US dollars (2007 rates). *Cost estimate was based on the CPT code 49425, insertion of peritoneal venous shunt. Assumptions included a two-day length of stay in general ward at \$725/day and two days of physician inpatient visits (CPT 99222 and CPT 99232). Unit costs: Ingenix for CPT codes, ${ }^{8,9}$ Putnam and colleagues for inpatient chest tube and pleural catheter, ${ }^{10}$ Girardi and colleagues for inpatient pericardial window, ${ }^{11}$ and Red Book for drug costs. ${ }^{12}$ 
We determined the unit cost per health care resource utilized and calculated the average per patient cost of pleural effusion with dasatinib. The average per patient cost of treating pleural effusions in this patient population was estimated by multiplying the number of units of health care resource use consumed by the probability of each event occurring. For example, in Table 2 the cost of a course of diuretics was estimated at $\$ 8$. The number of units was assumed to be 1.29 , taking into account the first occurrence and a $29 \%$ rate of recurrence of pleural effusion that would require additional treatment. The probability that a patient would require diuretic therapy was $68 \%$, as based on the literature. Therefore, the expected cost of diuretic treatment per patient was $\sim \$ 7$ ( $\$ 8$ cost of drug $\times 1.29$ units $\times 0.68$ probability). The expected costs for each health care resource use that may have occurred for all patients were calculated, as well as the average per patient cost considering all health care resources consumed. All grades of pleural effusion were considered in our analysis.

Sensitivity analyses were conducted to test the assumptions and impact on overall costs. All costs are presented in US dollars (2007 rates) to reflect year of clinical/resource data reported by the cancer center.

\section{Results}

Pleural effusion occurred in 35\% of dasatinib-treated patients. ${ }^{6,7}$ Sixty percent of pleural effusions reported at the cancer center involved $\leq 25 \%$ of one lung volume and were managed medically including diuretics and steroids. ${ }^{6,7}$ Costs for this medically managed group were $\$ 750$ per episode, including physician visits, $\mathrm{ECHO}$, chest X-rays and medications.

Forty percent of pleural effusions were more significant, involving $26 \%$ to $>75 \%$ of one lung volume, with half of those patients requiring invasive procedures. Forty-eight percent of all pleural effusions were characterized as NCI grade 3 or 4 based on symptoms. Inpatient procedures for placement of a chest tube or a pleural catheter had estimated costs of $\$ 10,616$ and $\$ 15,170$, respectively. The cost of invasive outpatient management of pleural effusions ranged from $\$ 713$ for ultrasound thoracentesis to $\$ 4,598$ for placement of a pleural catheter (see Table 2). Other costs of invasive procedures ranged from $\$ 3,807$ for placement of a Denver shunt (due to recurrent pleural effusions), to $\$ 15,344$ for patients who required a pericardial window (due to co-existing pericardial effusion with pleural effusion). Table 2 summarizes the unit cost per health care resource utilization associated with the treatment of pleural effusions.
The average per patient cost for treatment of pleural effusion was $\$ 2,062$. If patients were to have placement of a pleural catheter instead of thoracentesis or chest tube placement, the average per patient cost would increase to $\$ 2,717$. The average total cost of invasive procedure treatment for patients without a pleural catheter was $\$ 6,394$; this increased to $\$ 9,013$ in patients with a pleural catheter (Table 2).

In sensitivity analyses, alternating assumptions indicated that the analysis was robust. Costs of treatment of pleural effusions were changed based on Medicare reimbursement rates for various CPT codes, instead of median fees for private payers. Using Medicare reimbursements, the average cost per event decreased by only $6 \%-12 \%$ relative to the base case results. Assuming no recurrence of pleural effusion reduced total average costs by $\sim 20 \%$, while placement of a pleural catheter instead of repeated thoracentesis or chest tube placement increased the cost by $\sim 30 \%$. Additionally, excluding the cost for ultrasound-guided thoracentesis would only minimally decrease the average per patient costs of treating pleural effusions.

\section{Discussion}

This economic analysis based on observed treatment patterns suggests that the management of pleural effusions in CML requires medical intervention and is costly, particularly for those requiring invasive procedures. The total average cost per patient across all pleural effusions is about $\$ 2,000$ to $\$ 2,700$ depending on the type of intervention, while the total average cost per patient for those requiring invasive procedures is estimated to be $\$ 6,400$ to $\$ 9,000$. The rate of recurrence of pleural effusions was a major cost driver in this analysis. Each successive treatment of recurrent pleural effusion adds to the costs of therapy, with increased costs due to additional hospital stays, increased laboratory and radiology costs, increased personnel costs for procedures, and increased pharmacy costs. The rate of recurrence of pleural effusion was $29 \%$, based on the real-world rates reported in the literature. ${ }^{6}$

The cost of pleural effusion adverse events should be considered in the context of the total cost of treating the CML patient, of which the primary driver is currently the drug cost of tyrosine kinase inhibitor (TKI) therapy. Considering that the cost of the newer TKIs may be $>\$ 150$ per day, the cost of pleural effusions alone, averaged across the CML population, is not a major cost driver of the total cost of care. However, for the physician managing an individual patient with a significant pleural effusion requiring inpatient 
procedures, the pleural effusion may represent $>25 \%$ of the cost of treating their CML.

Development of pleural effusions with dasatinib poses a significant challenge to physicians as the risk persists over time, onset is unpredictable, and management may require repeat invasive procedures and possible complications. ${ }^{6,7}$ For example, twelve percent of dasatinib patients with CML-CP experience pleural effusions as late as 24 months into treatment. ${ }^{7}$ Over time, risk of all grade (and grade $3 / 4$ ) pleural effusions increases to $29 \%$ (12\% grade $3 / 4)$ in chronic phase and $50 \%$ (28\% grade $3 / 4)$ in accelerated phase. The true incidence of dasatinib-related pleural effusions in CML patients is not yet known because it is a relatively new treatment. However, the incidence of pleural effusion does differ based on the dosing regimen of dasatinib. Recent six-month phase III trial data published for the $100 \mathrm{mg}$ daily dose of dasatinib in CML-CP patients indicate lower rates of pleural effusion than the $140 \mathrm{mg}$ daily dose $(7 \%-10 \%$ vs $18 \%-20 \%$, respectively). ${ }^{13}$

While pleural effusion rates have been reduced with a $100 \mathrm{mg}$ daily dose of dasatinib, pleural effusion in CML remains a significant adverse event regardless of the dose and may be a clinically important factor in treatment selection. A frequent intervention in the management of pleural effusion is temporary interruption of therapy, which was required in $83 \%$ of dasatinib patients with a pleural effusion for a median of 27 days, after which the dose was reduced in $71 \% .{ }^{6,7}$ Almost one-third of patients required treatment interruptions on more than one occasion for recurrent pleural effusion; a total of $6 \%$ of patients were permanently discontinued from dasatinib for recurrent effusions. ${ }^{7}$ These patients may then be at risk for disease progression while the drug is being held or discontinued, and the broader socioeconomic cost of not effectively controlling CML during these pleural effusion events was not considered in the current analysis.

Potential limitations were addressed when possible through conservative approaches to the assumptions and conducting sensitivity analyses to assess main drivers of cost. While health care resource utilization data were based on pleural effusions occurring at one major US cancer center, the costs applied to the resources were general reimbursements or literature-based costs and not specific to that cancer center. Thus, because patient demographics and treatment patterns may differ among institutions, costs of treating pleural effusions may also vary. For example, we attempted to account for differences in treatment patterns by including the cost of both inpatient and outpatient pleural catheter placement in the analysis. ${ }^{10}$ We also used the median fees for various outpatient procedures, thus the cost estimates for half of the payers could be higher. Additionally, as a sensitivity analysis, a Medicare reimbursement perspective was considered and average cost of pleural effusion treatment decreased only somewhat, suggesting that regardless of type of reimbursement structure, treatment of pleural effusions remains costly. The most sensitive cost driver in the analysis was the type of invasive procedure selected for managing the more significant pleural effusions. If the physician is able to anticipate early on that multiple thoracentesis procedures will be required for a patient, use of an outpatient pleural catheter could reduce total cost of management by up to $30 \%$. Finally, due to gaps in the literature and variations in expert opinion, our analysis included conservative assumptions for additional office visits and X-rays, and did not include the cost of platelet transfusions that may be needed to perform thoracentesis or the cost of follow-up diagnostics/scans. Thus, while we present a conservative analysis, the results provide an initial estimate of the costs of treating pleural effusions in CML patients.

\section{Conclusions}

This economic analysis, based on observed treatment and resource use patterns, suggests that the management of pleural effusions in CML patients is costly and requires intensive resource utilization. Understanding of the clinical management and economic burden of treatment-related adverse events for patients with CML is essential in an era of tightening health care budgets. CML therapies without significant risk of pleural effusion may provide cost offsets related to adverse event management.

\section{Disclosure}

This research was funded by Novartis Pharmaceuticals.

\section{References}

1. Druker BJ, Guilhot F, O’Brien SG, et al. Five-year follow-up of patients receiving imatinib for chronic myeloid leukemia. $N$ Engl J Med. 2006;355(23):2408-2417.

2. Weisberg E, Manley PW, Cowan-Jacob SW, Hochhaus A, Griffin JD. Second generation inhibitors of BCR-ABL for the treatment of imatinibresistant CML. Nature Rev Cancer. 2007;7(5):345-356.

3. Bristol-Myers Squibb Company. Sprycel (dasatinib) prescribing information. Princeton, NJ: Bristol-Myers Squibb Company; 2008.

4. Novartis Pharmaceuticals. Tasigna (nilotinib) prescribing information. Florham Park, NJ: Novartis Pharmaceuticals; 2007.

5. Koledin M, Duric D, Milovancev A, Bijelovic M, Baros B. Pleural effusions of malignant etiology: diagnostics, treatment and quality of life. Arch Oncol. 2001;9(1):3-7.

6. Quintas-Cardama A, Kantarjian H, O’Brien S, et al. Pleural effusion in patients with chronic myelogenous leukemia treated with dasatinib after imatinib failure. J Clin Oncol. 2007;25(25):3908-3914. 
7. Quintas-Cardama A, Kantarjian HM, Munden R, et al. Pleural effusion in patients (pts) with chronic myelogenous leukemia (CML) treated with dasatinib after imatinib failure (abstract). ASH Annual Meeting. Blood. 2006;1:108(11):2164.

8. Ingenix. National Fee Analyzer: Charge data for evaluating fees nationally. Eden Prairie, MN: Ingenix; 2006.

9. Ingenix. Outpatient billing expert. Eden Prairie, MN: Ingenix; 2006.

10. Putnam JB Jr, Walsh GL, Swisher SG, et al. Outpatient management of malignant pleural effusion by a chronic indwelling pleural catheter. Ann Thorac Surg. 2000;69(2):369-375.
11. Girardi LN, Ginsberg RJ, Burt ME. Pericardiocentesis and intrapericardial sclerosis: effective therapy for malignant pericardial effusions. Ann Thorac Surg. 1997;64(5):1422-1427.

12. Thomson Healthcare. Red Book. Montvale, NJ: Thomson Healthcare; 2008.

13. Shah NP, Kantarjian HM, Kim DW, et al. Intermittent target inhibition with dasatinib $100 \mathrm{mg}$ once daily preserves efficacy and improves tolerability in imatinib-resistant and -intolerant chronic-phase chronic myeloid leukemia. J Clin Oncol. 2008;26(19):3204-3212.

\section{Publish your work in this journal}

The International Journal of General Medicine is an international, peer-reviewed open-access journal that focuses on general and internal medicine, pathogenesis, epidemiology, diagnosis, monitoring and treatment protocols. The journal is characterized by the rapid reporting of reviews, original research and clinical studies across all disease areas.
A key focus is the elucidation of disease processes and management protocols resulting in improved outcomes for the patient. The manuscript management system is completely online and includes a very quick and fair peer-review system. Visit http://www.dovepress.com/ testimonials.php to read real quotes from published authors. 